\title{
Proliferating Trichilemmal Tumor - A Very Rare Disease In Young Adults
}

\author{
Diana Monteiro $^{1 *}$, Jorge Pinheiro ${ }^{2}$, Pedro Silva ${ }^{1}$, Álvaro Silva ${ }^{1}$ \\ ${ }^{1}$ Department of Plastic, Reconstructive and Aesthetic and Burn Unity, Faculty of Medicine of Porto University, Alameda Professor Hernâni Monteiro, $4200-319$ \\ Porto, Portugal \\ ${ }^{2}$ Department of Pathology, Centro Hospitalar São João, Faculty of Medicine of Porto University, Alameda Professor Hernâni Monteiro, $4200-319$ Porto, \\ Portugal
}

Received: 09 August, 2016; Accepted: 16 August, 2016; Published: 26 August, 2016

*Corresponding author: Diana Isabel Moreira Monteiro, Department of Plastic, Reconstructive and Aesthetic and Burn Unity, Faculty of Medicine of Porto University, Alameda Professor Hernâni Monteiro, Rua da Rasa, no 119, 4o Esquerdo, 4400-271 Vila Nova Gaia, Portugal, Tel: +351.93.8232590; E-mail: dimmonteiro@gmail.com

\begin{abstract}
Proliferating Trichilemmal Tumor (PTT) is a very rare usually benign tumor found in the scalp and face of elderly females. However, at times, the tumor has aggressive clinical course and a propensity for nodal and distant metastases.

We report cases of PTT over the scalp on a very uncommon age.

Keywords: Cysts; Scalp neoplasms; Pilar tumor; Trichilemmal tumor; Trichilemmal keratinization
\end{abstract}

Trichilemmal Tumor (PTT), first recognized by Wilson-Jones 1966 [1] is a rare, usually benign tumor of Proliferating external root sheath derivation affecting scalp, eyelids, neck and face of elderly female patients. It have been reported with varying nomenclature, e.g., invasive pilomatrixioma, proliferating epidermoid cyst, trichoclamydocarcinoma and pilar tumor [1-2] It is a slow growing, large, solitary, multilobulated lesion that may arise within a pilar cyst. These tumors are largely benign, often cystic, and are characterized by trichilemmal keratinization. Tumors with an invasive growth pattern or cytologic atypia have an unpredictable course and may be locally aggressive or have a propensity for nodal and distant metastases. Thus, it has been suggested that even the classical PTT should be considered as carcinoma [2-3]. Due to its rarity, there are no guidelines available for the management of these tumors.

A 30-year-old woman (patient 1) and a 26-year-old pregnant woman (patient 2) presented with a swelling on the parietal and occipital region of the scalp, with diameters of 3 and $2.5 \mathrm{~cm}$, respectively. The lesions had evidently been growing in the last 3 months. There was no pain or erythematic or history of trauma or lymadenopathy in both patients. Their medical history was unremarkable. The nodular masses with a smooth surface and soft consistency, were not fixed to the underlying skull bone and periosteum. There were no palpable adenomegalies. Total lesion surgical excision with a $0.5 \mathrm{~cm}$ margin of normal tissue was performed under local anesthesia (Figure 1).
Hematoxylin-eosin stain revealed a multilobular and cystic tumor with pushing borders and lobulated contour, occupying the dermis, involving the epidermis and opening into skin surface (Figure 2A). Lobules displayed peripheral palisading of small basaloid cells, differentiating towards large keratinocytes with ample eosinophilic cytoplasm and abrupt keratinization without a granular layer - trichilemmal keratinization (Figure 2B). Tumor cells showed moderate nuclear atypia, nuclear pleomorphism and scarce mitoses (Figure 2C). This was consistent with PTT,

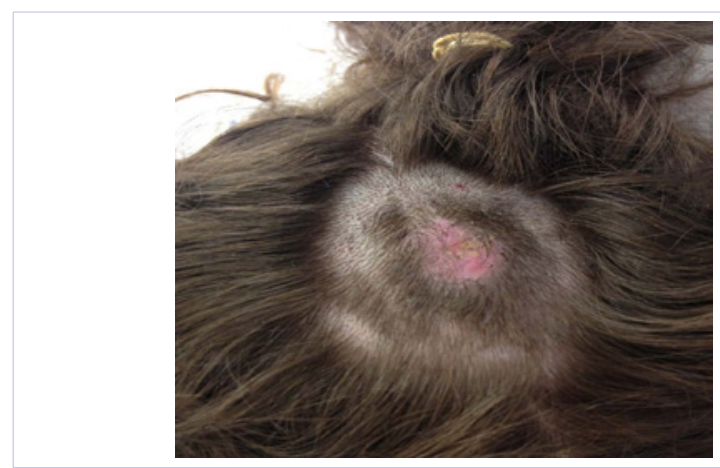

Figure 1: Asymptomatic nodule on the sclap (patient 2)

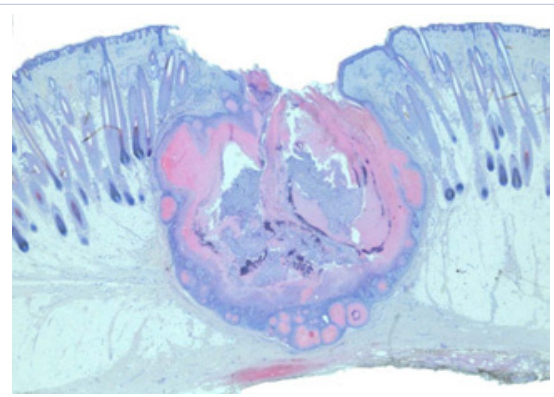

Figure 2a: Multilobular and cystic tumor (patient $1 \mathrm{H \& E}, \mathrm{x} 4$ ) 
so they underwent surgical reintervention to wide local excision with $1 \mathrm{~cm}$ margin of normal tissue and no relapse was observed during the 1-year period of follow-up.

PTT is more frequent in middle-aged or elderly female patients and more than $90 \%$ of the lesions are localized on the scalp but the face, trunk, back, wrist and vulva can also be affected. Etiopathogenesis remains unknown but in most cases it appears to developed within the wall of a pre-existenting pilar cyst as complication of trauma and inflammation. It has also been reported to develop in nevus sebaceous and human papillomavirus has also been implicated [2]. Hence, patients frequently give history of a long standing cyst at the same site. Nevertheless, PTT can occur de novo, in young adults, as in our report. Histologically, PPT is made up of massively proliferating lobules of squamous epithelium showing multiple central areas of trichilemmal keratinization and formation of homogenous keratin cysts. Brownstein et al concluded that the most characteristic feature of PTTs was trichilemmal keratinization [3].

Accordingly, Ye et al. [4] classified PTTs into three groups, namely, benign, low-grade malignant, and high-grade malignant lesions, based on clinicopathological characteristics. Group I PTTs are benign lesions. Recurrences are not observed and histologically show regular contours with surrounding tissues and mild nuclear atypia but do not involve increased mitotic activity, necrosis, and lymphovascular invasion. Group II PTTs are low-grade malignant tumors. Local recurrence may be observed in these lesions. These tumors have histologically irregular and local invasive contours, and they elongate to the deep dermis and subcutaneous tissue. Group III PTTs are high-grade malignant

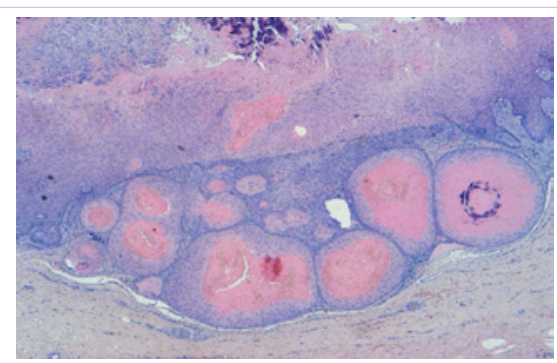

Figure 2b: Peripheral palisading of small basaloid cells, differentiating towards large keratinocytes and abrupt trichilemmal keratinization (patient $1 \mathrm{H} \& \mathrm{E}, \mathrm{x}$ 10)

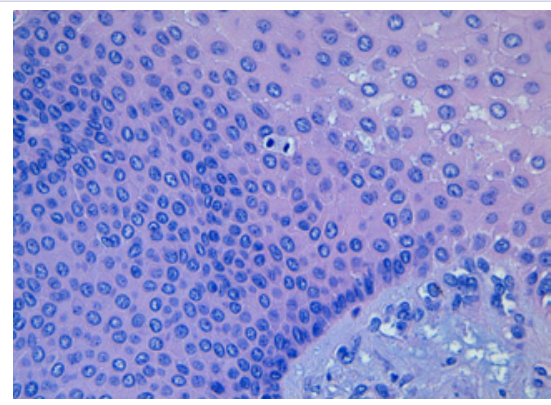

Figure 2c: Mild nuclear atypia and scarce mitoses (patient $1 \mathrm{H} \& \mathrm{E}, \mathrm{x} 20$ ) tumors reported to exhibit a high recurrence rate, lymph nodes, and tendency to develop distant metastasis. Remarkable nuclear pleomorphism, atypical mitosis, necrosis, and lymphovascular invasion may be observed in these tumors. On the basis of this classification, our cases were classified as benign lesions because of regular contours with surrounding tissue, the generally mild degree of atypia and pleomorphism, aside from the absence of lymphovascular invasion and necrosis and absence of a local or distant organ metastasis and recurrence despite a 12-month follow-up, which clinically supports this conclusion.

It is accepted that cytologic atypia may be present in PTT that ultimately have a benign behaviour. However, the malignant variant also shows cytologic atypia and mitotic activity. This issue remains controversial since tumors with little or no cytologic atypia may be aggressive, showing that their histologic appearance does not necessarily correlate with their biological behavior [5]. Cases with good prognosis despite remarkable cytological atypia, as well as patients who died because of metastatic disease, although they did not present an inltrative growth pattern, have been reported in the literature.

Therefore, as proposed by some authors, a nonscalp location, recent rapid growth, size larger than $5 \mathrm{~cm}$, infiltrative growth and important cytologic atypia with mitotic activity should be regarded as malignancy [4-5]. Infiltrating tumors with marked atypia and pleomorphism may be histologically mistaken as Squamous Cell Carcinoma (SCC) and Trichilemmal Carcinoma (TC). Trichilemmal-type keratinization, calcification, eosinophilic hyaline membrane surrounding the tumor, ordinary trichilemmal cyst, lack of a precursory epidermal lesion are all indicators of PTT rather than SCC. It is important to distinguish TC from the malignant variant of PTT, because the first does not have metastatic potential. TC is centered around a pilosebaceous unit and is composed of cytologically atypical, glycogen-containing or pale clear cell with basilar or full-thickness interfollicular epidermal spread; there is always a connection to the epidermis [4-5].

In summary, these are two cases of a rare tumor on an uncommon age, with uncertain clinic behaviour and prognosis, which may represent a challenge because there are no guidelines available for the management of these tumors. Another issue is that the morphological characteristic of PTTs may not always be compatible with the clinical presentation. Therefore, complete local excision with one-centimeter margin of normal tissue and a long-term clinical follow-up is still the standard treatment. Considering the aggressive nature of the malignant variant in reported series adjuvant chemotherapy and radiotherapy have been used to prevent recurrence.

Conflict of interests: The authors declare that there is no conflict of interests regarding the publication of this paper.

\section{References}

1. EW Jones. Proliferating epidermoid cysts. Archives of Dermatology. 1966; 94(1):11-19. doi:10.1001/archderm.1966.01600250017002. 
2. Takeda H, Ikenaga S, Kaneko T, Nakajima K, Harada K, Hanada K, et al. Proliferating trichilemmal tumor developing in nevus sebaceous. Eur J Dermatol. 2010;20(5):664-5. doi: 10.1684/ejd.2010.1044.

3. Brownstein MH, Arluk DJ. Proliferating trichilemmal cyst: a stimulant of squamous cell carcinoma. Cancer. 1981;48(5):1207-1214.

4. Ye J, Nappi O, Swanson PE, Patterson JW, Wick MR. Proliferating pilar tumors. A clinicopathologic study of 76 cases with a proposal for definition of benign and malignant variants. Am J Clin Pathol. 2004;122(4):566-574.

5. Folpe AL, Reisenauer AK, Mentzel T, Rütten A, Solomon AR. Proliferating trichilemmal tumors: clinicopathologic evaluation is a guide to biologic behavior. J Cutan Pathol. 2003;30(8):492-498. 